\title{
Analysis of aberrant virulence of Gibberella zeae following transformation-mediated complementation of a trichothecene-deficient (Tri5) mutant
}

\author{
Anne E. Desjardins, ${ }^{1}$ Gui-hua Bai, ${ }^{2}$ Ronald D. Plattner ${ }^{1}$ \\ and Robert $\mathrm{H}$. Proctor ${ }^{1}$
}

1 Mycotoxin Research Unit, National Center for Agricultural Utilization Research, United States Department of Agriculture, Agricultural Research Service, Peoria, IL 61604, USA

2 Department of Plant and Soil Science, Oklahoma State University, Stillwater, OK 74078, USA

\author{
Author for correspondence: Anne E. Desjardins. Tel: +1 309681 6378. Fax: +1 3096816671 . \\ e-mail: desjarae@mail.ncaur.usda.gov
}

\begin{abstract}
Gibberella zeae causes wheat ear blight and produces trichothecene toxins in infected grain. In previous studies, trichothecene production in this fungus was disabled by specific disruption of the trichodiene synthase gene (Tri5) and was restored by two methods: gene reversion and transformation-mediated mutant complementation. In previous field tests of wheat ear blight, trichothecene-nonproducing mutants were less virulent than the wild-type progenitor strain from which they were derived. Trichothecene-producing revertants also were restored to wild-type levels of virulence. In contrast, in the field test of wheat ear blight reported here, trichothecene-producing strains obtained by Tri5 mutant complementation were not restored to wildtype levels of virulence. The complemented mutants showed a slightly reduced radial growth compared to the wild-type strain, but otherwise appeared normal in morphology, pigmentation and sexual fertility. Genetic analysis indicated that the aberrant virulence of a complemented mutant was likely due to non-target effects that occurred during the process of transforming the trichothecene-nonproducing mutant with Tri5. These results confirm previous findings that trichothecenes contribute to the virulence of G. zeae, but also demonstrate that manipulating this fungus in the laboratory may cause it to undergo subtle changes that reduce its virulence.
\end{abstract}

Keywords: Gibberella zeae, Fusarium graminearum, trichothecene toxins, wheat ear blight

\section{INTRODUCTION}

Gibberella zeae (anamorph Fusarium graminearum) causes ear and seedling blights (scab) of wheat and other small grain cereals and produces trichothecene mycotoxins in infected grain (Bai \& Shaner, 1994; Parry et al., 1995). This fungus also infects maize ears and stalks and a diversity of other plants and plant tissues worldwide (Sutton, 1982). Trichothecenes are highly toxic to plants and are harmful to human and animal health upon ingestion (Desjardins et al., 1993). Historical and epidemiological data have associated human disease outbreaks in Eastern Europe and in Asia with the consumption of grain contaminated with trichothecenes (Marasas et al., 1984). Despite the economic importance of G. zeae, its mechanisms of pathogenesis have received little attention. Although a diversity of virulence mech- anisms undoubtedly exists, to our knowledge the only virulence factor that has been established for G. zeae is the production of trichothecene toxins. The role of trichothecenes in the ability of $G$. zeae to cause wheat ear and seedling blight and maize ear rot has been investigated using trichothecene-nonproducing mutants obtained by transformation-mediated disruption of the trichodiene synthase gene (Tri5) using hygromycin phosphotransferase $(h y g B)$ as a selectable marker. The enzyme trichodiene synthase catalyses the cyclization of the isoprenoid farnesyl pyrophosphate to trichodiene, which is the first unique step in the trichothecene biosynthetic pathway. As trichodiene synthase is present as a single copy in this haploid fungus, G. zeae mutants lacking a functional Tri5 are unable to produce trichothecenes (Proctor et al., 1995).

Trichothecene-nonproducing mutants of G. zeae were 
initially characterized for the ability to cause ear and seedling blights on wheat plants in a growth chamber (Proctor et al., 1995). The virulence of two Tri5 disruption mutants was then tested on wheat ears and maize ears under field conditions (Desjardins et al., 1996; Nicholson et al., 1998; Harris et al., 1999). In all of the virulence tests in growth chambers and in the field, trichothecene-nonproducing mutants produced less disease on wheat and maize than the trichotheceneproducing progenitor strain from which they were derived. Although transformation-mediated gene disruption is a powerful tool for examining the role of genes in pathogenesis, the transformation process itself can cause alterations at loci other than the targeted gene. Consequently, a change in virulence associated with Tri5 disruption may not be sufficient proof that Tri5 is involved in pathogenesis of G. zeae.

Two major approaches have been used to determine whether the reduced virulence of Tri5 mutants of G. zeae is due specifically to disruption of the gene or to non-target effects resulting from the transformation process. In the first approach, a revertant was generated by allowing a Tri5 disruption mutant to pass through the sexual phase of its life cycle. In one of the resulting progeny, the disrupted Tri5 had reverted to wild-type and the strain regained the ability to produce trichothecenes and to produce seedling blight on wheat plants in a growth chamber test (Proctor et al., 1997). To facilitate strain tracking during field tests, this revertant was tagged by transforming it with a vector containing the $h y g B$ gene. Two of the resulting transformants were tested for virulence on wheat ears and maize ears under field conditions. Both tagged revertants were restored to wild-type levels of virulence in field tests (Desjardins et al., 1996; Nicholson et al., 1998; Harris et al., 1999). In the second approach, a Tri5 disruption mutation was complemented by introducing a functional copy of Tri5 by a second transformation protocol using acetamidase $(a m d S)$ as a selectable marker. In some of these transformants, the ability to produce trichothecenes was restored. Two of these trichothecene-producing complemented mutants were tested for virulence on wheat seedlings in a growth chamber, and both were restored to near wild-type levels of virulence (Proctor et al., 1997).

The present study was carried out to further evaluate the importance of trichothecenes in virulence of G. zeae by testing three trichothecene-producing complemented mutants on wheat ears under field conditions. Unexpectedly, the complemented mutants showed little or no increase in virulence on wheat ears compared to the trichothecene-nonproducing mutant from which they were derived. To investigate this anomalous result, we conducted transformation experiments to determine the effect of the second transformation protocol on virulence of the wild-type trichothecene-producing progenitor strain. We also outcrossed the original Tri5 disruptant and one of the complemented mutants with the wild-type progenitor strain, then analysed the resulting meiotic progeny for segregation of the intro- duced genes and for virulence. These analyses indicated that the reduced virulence of the complemented mutants was likely due to non-target effects that occurred during the process of transforming the trichothecenenonproducing mutant with Tri5.

\section{METHODS}

Fungal strains. The trichothecene-producing wild-type progenitor strain GZ3639, the trichothecene-nonproducing Tri5 disruption strain GZT40 derived from GZ3639, and the trichothecene-producing Tri5 complemented strains GZT2011, GZT201-2 and GZT201-5 derived from GZT40 have been previously described (Proctor et al., 1997). Accession numbers of strains GZ3639 and GZT40, respectively, are NRRL22917 and NRRL-22916, National Center for Agricultural Utilization Research, Peoria, IL, USA. Tri5 genotypes of the strains are given in Table 1 . All cultures and crosses were grown under continuous illumination at $20 \pm 1{ }^{\circ} \mathrm{C}$ provided by an equal mixture of fluorescent white and black (General Electric BLB, $40 \mathrm{~W}$ ) light bulbs. Strains were routinely cultured on V8 juice agar medium (V8 juice, $200 \mathrm{ml} ; \mathrm{CaCl}_{2}, 3 \mathrm{~g}$; and agar, $20 \mathrm{~g} \mathrm{l}^{-1}$ ). Strains were also evaluated for growth and morphology on plates of potato dextrose agar medium (Nelson et al., 1983) and minimal agar medium (Correll et al., 1987). Radial growth rates and sporulation on minimal medium were determined from four replicate plates of each strain. Conidia for virulence assays were usually harvested from strains grown in mung bean liquid medium for $4 \mathrm{~d}$ at $28^{\circ} \mathrm{C}$ (Bai \& Shaner, 1996). If necessary, mung bean cultures were stored at $4{ }^{\circ} \mathrm{C}$ for up to $10 \mathrm{~d}$ prior to use.

Crosses. Spontaneous nitrate-nonutilizing (nit) mutants were generated on minimal medium supplemented with $1.5 \%(\mathrm{w} / \mathrm{v})$ chlorate and their phenotypes were scored on basal medium amended with different nitrogen sources (Bowden \& Leslie, 1992). Crosses were made by placing two strains on opposite sides of a $60 \mathrm{~mm}$ Petri plate containing carrot agar (Bowden $\&$ Leslie, 1999). After one week, $2 \mathrm{ml}$ sterile water was added to the culture and spread with a plastic loop to flatten the mycelium. Cultures were incubated for an additional 2-4 weeks until cirrhi were observed oozing from perithecia. To obtain single ascospore strains, individual cirrhi were picked from the interface of the two parental colonies and the ascospores were suspended by vortexing each cirrhus in $2 \mathrm{ml}$ sterile water. The resulting suspension was poured over a plate of $5 \%(\mathrm{w} / \mathrm{v})$ water agar and incubated for 16-20 h. With the aid of a stereomicroscope, small blocks of agar were cut around individual germinating ascospores and transferred to minimal medium. All progeny from perithecia that contained prototrophs were reisolated from single conidia and analysed for segregation of Tri5 alleles by PCR as described below. Selected progeny were also scored for nit mutant phenotype as described above.

Transformation of strain GZ3639. Protoplasting and transformation procedures with vector pADD6-1 were carried out as described previously (Proctor et al., 1997). Aliquots of the transformation mixture were spread on basal medium supplemented with $10 \mathrm{mM}$ acetamide. Transformation plates were incubated in the dark at $28{ }^{\circ} \mathrm{C}$ and transformants were detected after 1 week. Putative transformants were transferred to V8 juice agar slants for storage, and selected transformants were reisolated from single spores for further analysis.

Field virulence test. The field test described in this report was conducted under permit granted by the United States De- 
Table 1. Disease ratings, yield loss and deoxynivalenol and ergosterol content of wheat ears inoculated in the field with G. zeae

Numbers in each column followed by the same letter are not significantly different at $P \leqslant 0 \cdot 05$.

\begin{tabular}{|c|c|c|c|c|c|c|}
\hline \multirow[t]{2}{*}{ Strain } & \multirow[t]{2}{*}{ Genotype* } & \multicolumn{2}{|c|}{ Disease rating $(\%) \dagger$} & \multirow[t]{2}{*}{ Yield $(\mathrm{g}) \neq$} & \multirow{2}{*}{$\begin{array}{c}\text { Deoxynivalenol } \\
\left(\mu \mathrm{g} \mathrm{g}^{-1}\right) \mathbb{S}\end{array}$} & \multirow{2}{*}{$\begin{array}{c}\text { Ergosterol } \\
\left(\mu \mathrm{g} \mathrm{g}^{-1}\right) \|\end{array}$} \\
\hline & & $\begin{array}{l}\text { Blighted } \\
\text { spikelets }\end{array}$ & $\begin{array}{l}\text { Scabby } \\
\text { kernels }\end{array}$ & & & \\
\hline GZ3639 & Tri5-1 & $84^{\mathrm{a}}$ & $42^{\mathrm{a}}$ & $15^{\mathrm{d}}$ & $51^{\mathrm{a}}$ & $54^{\mathrm{a}}$ \\
\hline GZT201-1 & Tri5-3/Tri5-4 & $20^{\mathrm{b}}$ & $10^{\mathrm{cd}}$ & $56^{\mathrm{ab}}$ & $14^{\mathrm{d}}$ & $13^{\mathrm{b}}$ \\
\hline GZT201-2 & Tri5-3/Tri5-4 & $25^{\mathrm{b}}$ & $16^{\mathrm{bc}}$ & $46^{c}$ & $35^{\mathrm{c}}$ & $19^{b}$ \\
\hline GZT201-5 & Tri5-3/Tri5-4 & $28^{\mathrm{b}}$ & $14^{\mathrm{cd}}$ & $47^{c}$ & $44^{\mathrm{b}}$ & $20^{\mathrm{b}}$ \\
\hline GZT40 & Tri5-3 & $25^{\mathrm{b}}$ & $13^{\mathrm{cd}}$ & $54^{\mathrm{b}}$ & $12^{\mathrm{d}}$ & $20^{\mathrm{b}}$ \\
\hline Water & & $24^{\mathrm{b}}$ & $8^{\mathrm{d}}$ & $59^{\mathrm{a}}$ & $10^{\mathrm{d}}$ & $9^{c}$ \\
\hline
\end{tabular}

*Tri5-1 is the wild-type allele; Tri5-3 is the disrupted allele; Tri5-4 is the complementing allele.

† Mean percentage of blighted spikelets per ear in the field; mean percentage of scabby kernels by weight in the harvested kernels.

$\ddagger$ Mean kernel weight per 100 ears.

$\$$ Mean amount of deoxynivalenol $\left[\mu \mathrm{g}(\mathrm{g} \text { kernel dry wt })^{-1}\right]$ determined by fluorometric assay.

$\|$ Mean amount of ergosterol $\left[\mu \mathrm{g}(\mathrm{g} \text { kernel dry wt })^{-1}\right]$ determined by HPLC.

partment of Agriculture, Animal and Plant Health Inspection Service. Hard red spring wheat cultivar Wheaton, which is highly susceptible to ear blight, was hand-sown on 12 April 1998, at the Walter Christ Farm 24 km west of Peoria, Illinois. The test was a randomized complete block design with three replications. Four rows of wheat, approximately 100 seeds per row, were planted in each of the 18 plots. Each plot was $1 \times 1 \mathrm{~m}$, and was surrounded by cleared alleys that were approximately $1 \mathrm{~m}$ wide. The entire test was surrounded by a 1-m-wide border of wheat plants, then by a 10-m-wide border that was cleared of all vegetation.

When the ears reached the mid-anthesis stage of growth, 100 ears in each plot were tagged with coloured tape just below the ear. Approximately 25 ears were tagged in each of the four rows in each plot. On 8, 9 and 10 June the tagged ears were inoculated by injecting a drop of conidium suspension at $10^{5} \mathrm{ml}^{-1}$ into one floret of a central spikelet. This method of inoculation delivered approximately $20 \mu \mathrm{l}$ inoculum containing $2 \times 10^{3}$ conidia. Control plants were inoculated with sterile water. Disease was assessed as the percentage of blighted spikelets per ear $20 \mathrm{~d}$ after inoculation. Ears were harvested at maturity on 13 and 14 July and threshed individually, and the total kernels and the scabby kernels of each ear were counted and weighed. After individual ears were assessed, one kernel was removed from the centre of each of 10 representative ears from each plot for microbiological tests. The remaining kernels from each plot were assigned to two bulked pools per plot: pool A of each plot contained kernels from ears 1-50, and pool B of each plot contained kernels from ears 51-100. The bulked kernels were ground and stored at $-20^{\circ} \mathrm{C}$ until they were analysed for trichothecenes and ergosterol.

Identification of fungal strains by PCR. Kernels sampled from inoculated ears were surface-disinfested with $0.5 \%(\mathrm{w} / \mathrm{v})$ sodium hypochlorite and cultured on a Fusarium selective medium containing pentachloronitrobenzene (Nelson et al., 1983). Putative G. zeae colonies were transferred to potato dextrose agar to facilitate identification, then subcultured to individual plates of V8 juice agar medium. Transformants and ascospore progeny also were subcultured to plates of V8 juice agar medium. Mycelium harvested from the plates was lyophilized, and fungal genomic DNA was isolated as previously described (Proctor et al., 1997), except that after phenol/chloroform extraction, the DNA was purified further using an UltraClean DNA Purification kit (MoBio Laboratories). The resulting DNA was used as template for PCR reactions as previously described (Proctor et al., 1997) with primer pairs capable of specifically identifying various genes. The Tri5-1 allele was identified using primers 1099 and 1100, which bind within the Tri5 coding region near the $5^{\prime}$ and $3^{\prime}$-ends, respectively, and amplify a 985 bp PCR product. The Tri5-3 allele was identified using primer 1098, which binds near the $3^{\prime}$-end of the $h y g B$ gene. Primers 1098 and 1100 amplify a 688 bp PCR product. The amdS gene was identified using primers 880 and 881 , which bind within the amdS coding region and amplify a 747 bp PCR product. The PCR product band pattern from each fungal strain was compared to those from the wild-type strain and mutant strains to determine its genotype. The sequences of the PCR primers were as follows: 880, 5'-GGGACTCGGTTCTGACAACC-3' ; 881, 5' -CCGAAATCGTGCTTGTATGG-3'; 1098， 5'-ACCAAGCCTATGCCTACAGCATCC-3'; 1099, 5' -AAGGTAGACCCTAAGCGACTACAG-3'; 1100, 5' -CTTCTGAGCCTCCTTCACATCGTC-3'.

Chemical analysis of grain from the field test. For trichothecene analysis, one $10 \mathrm{~g}$ sample of each ground, bulked pool $A$ and $B$ of kernels from the plots was extracted and analysed for deoxynivalenol using a fluorometric quantitation method (The Fluoroquant D Test kit; Romer Laboratories). When sufficient sample was available, two samples of each pool were extracted and analysed, and the results were combined. The detection limit for deoxynivalenol was $1 \mu \mathrm{g} \mathrm{g}^{-1}$.

Ergosterol analysis was used to determine total fungal biomass. For ergosterol analysis, samples of the ground, bulked pools $\mathrm{A}$ and $\mathrm{B}$ of kernels from the plots were saponified 
and extracted with hexane as previously described (Schwadorf \& Muller, 1989). Extracts were analysed by HPLC on a Spectra Physics SP8800 system with a C18 reversed phase column (Alltech), and a UV detector at $282 \mathrm{~nm}$. The presence and amount of ergosterol in the extracts were determined by comparing HPLC retention times and peaks with an ergosterol standard (Sigma). For all but one treatment group, one $10 \mathrm{~g}$ sample of each pool of kernels was extracted and analysed. Due to insufficient amounts of kernels treated with the wild-type strain GZ3639, only a $5 \mathrm{~g}$ sample of replicates 1 and 2, pools $\mathrm{A}$ and $\mathrm{B}$, was analysed, and replicate 3 could not be analysed.

Greenhouse virulence tests. Seedlings of cultivar Wheaton were grown for approximately 6 weeks in $18 \mathrm{~cm}$ plastic pots of pasteurized potting mixture (Redi-Earth Peat-lite mix; ScottsSierra Horticultural Products) in a controlled environment chamber at $15{ }^{\circ} \mathrm{C}$ with a $12 \mathrm{~h}$ light and a $12 \mathrm{~h}$ dark cycle. The seedlings were thinned to three to four plants per pot and the pots were moved to greenhouse benches with supplemental lighting. Greenhouse temperatures were set to $23^{\circ} \mathrm{C}$ during the day and $17^{\circ} \mathrm{C}$ during the night. During the course of the study, however, greenhouse temperatures occasionally varied significantly; from 19 to $32{ }^{\circ} \mathrm{C}$ during the day, and from 10 to $21{ }^{\circ} \mathrm{C}$ during the night. Plants were watered daily and fertilized weekly. Plants at the early anthesis stage were inoculated by injecting a drop of conidium suspension containing approximately $1 \times 10^{3}$ conidia into one floret of a central spikelet of each ear. A total of 10 ears in two to three pots were inoculated for each treatment. After inoculation the ears were covered with a plastic bag, which was left on for $3 \mathrm{~d}$. Virulence tests were conducted in five cycles from November 1998 through May 1999 and in November and December 1999. Due to poor symptom development in tests 4 and 5, ears were reinoculated 1 week after the first inoculation. Disease severity was calculated as the percentage of blighted spikelets in each ear at $18-21 \mathrm{~d}$ after the first inoculation in tests 1, 2, 3 and 5, and $18 \mathrm{~d}$ after the second inoculation in test 4 . Ears were harvested at maturity, usually 30-35 d after inoculation, and were threshed individually. The total kernels in each individual ear were counted and weighed. For deoxynivalenol analysis, kernels from 10 replicate ears of each treatment were pooled, ground and analysed by a fluorometric method as described above.

Statistical analysis. Data from plot means were analysed in the field experiment, and data from single ears were analysed in the greenhouse experiments. Analysis of variance was conducted on the field data in a random complete block experimental design. The treatments comprised five strains of G. zeae and a water-inoculated control, with three replicated plots per treatment. The dependent variables consisted of percentage blighted spikelets per ear, percentage scabby kernels per ear, yield as 100 ear weight in grams, deoxynivalenol level in kernels and ergosterol level in kernels. When significant $F$-statistic results were obtained, Fisher's LSD was used as the post-hoc multiple comparison test at the $5 \%$ level. Statistical analysis of field data and of greenhouse spikelet blight data was conducted according to Steel \& Torrie (1980) using StatView (Macintosh) programs (SAS Institute, Cary, NC). Greenhouse studies of percentage blighted spikelets per ear and of yield as 100 ear weight in grams were analysed by a one-way analysis of variance of G. zeae strains and a waterinoculated control. Fisher's LSD was the multiple comparison test used for blighted spikelets and Tukey's LSD using Statistix for Windows was used on yield data when significant Fstatistics were obtained at the $5 \%$ level.

\section{RESULTS}

\section{Aberrant virulence on wheat ears following complementation of a Tri5 mutant}

Virulence of $G$. zeae strains on wheat under field conditions was assessed by their ability to cause ear blight following inoculation of individual spikelets with conidia. Disease severity was assessed by determining the percentage of blighted spikelets per ear, and by analysing the harvested kernels for the percentage of scabby kernels and for yield loss. Kernels were also characterized for levels of deoxynivalenol and for fungal biomass as determined by ergosterol analysis. Field test data are presented in Table 1 . By all parameters tested, the Tri5 disruption (Tri5-3) mutant GZT40 caused significantly less $(P \leqslant 0 \cdot 05)$ disease than its wild-type (Tri5-1) progenitor, GZ3639. None of the trichothecene-producing Tri5-complemented (Tri5-3/Tri5-4) mutants, GZT201-1, GZT201-2 or GZT201-5, produced wild-type levels of disease by any of the parameters tested. In fact, the complemented mutant GZT201-1 was not significantly different from its progenitor strain, GZT40, in any of the disease parameters tested. In contrast, complemented mutants GZT201-2 and GZT201-5 caused significantly more yield loss than their progenitor strain, GZT40, and also produced moderate levels of deoxynivalenol in the infected ears. Frequent rain during anthesis was the probable cause of a relatively high incidence of natural infection and deoxynivalenol contamination in waterinoculated control ears.

Although the trichothecene-nonproducing mutant GZT40 and the complemented mutants GZT201-1, GZT201-2 and GZT201-5 were much less virulent than the wild-type strain, GZ3639, PCR analysis of strains from kernels harvested from the field test indicated that all four mutants retained the ability to survive in wheat ears. Mutant GZT40 was recovered at a frequency of $86 \%$ among 22 isolates of G. zeae recovered from ears inoculated with this mutant. Likewise, mutant GZT2011 was recovered at a frequency of $43 \%$ among 30 isolates; mutant GZT201-2 at a frequency of $67 \%$ among 30 isolates; and mutant GZT201-5 at a frequency of $47 \%$ among 30 isolates. No mutant strains were recovered from ears inoculated with the wild-type strain, GZ3639, or with water.

Virulence of G. zeae strains on wheat ears in the greenhouse was visually assessed by counting the percentage of blighted spikelets per ear following inoculation with conidia. In greenhouse tests 1, 2 and 3, ears were inoculated once; in tests 4 and 5 , ears were reinoculated 1 week after the first inoculation. In the greenhouse, strain GZ3639 produced a rapidly spreading lesion and extensive spikelet blight, whereas the mutant strains produced lesions that usually spread more slowly. Symptoms caused by mutant GZT40 usually were restricted to the single inoculated spikelet. The percentage of spikelets blighted by the wild-type strain GZ3639 was $85,85,97,99$ and $67 \%$ in tests 1,2 , 3,4 and 5, respectively. The percentage of spikelets 
Table 2. Disease ratings, yield loss and deoxynivalenol content of wheat ears inoculated in the greenhouse with vector pADD6-1 transformants of G. zeae strain GZ3639

\begin{tabular}{|llccc|}
\hline Strain & Genotype* & $\begin{array}{c}\text { Blighted } \\
\text { spikelets }(\%) \dagger\end{array}$ & $\begin{array}{c}\text { Yield } \\
(\mathbf{g}) \neq\end{array}$ & $\begin{array}{c}\text { Deoxynivalenol } \\
\left(\boldsymbol{\mu g} \mathbf{g}^{-1}\right) \mathbb{S}\end{array}$ \\
\hline GZ3639 & Tri5-1 & $92^{\mathrm{ab}}$ & $35^{\mathrm{d}}$ & 160 \\
GZT202-13 & Tri5-1/Tri5-4 & $100^{\mathrm{a}}$ & $29^{\mathrm{d}}$ & 150 \\
GZT202-10 & Tri5-1/Tri5-4 & $80^{\mathrm{ab}}$ & $83^{\mathrm{bc}}$ & 85 \\
GZT202-11 & Tri5-1/Tri5-4 & $62^{\mathrm{abc}}$ & $65^{\mathrm{bc}}$ & 75 \\
GZT202-1 & Tri5-1/Tri5-4 & $52^{\mathrm{bc}}$ & $50^{\mathrm{cd}}$ & 42 \\
GZT202-5 & Tri5-1/Tri5-4 & $51^{\mathrm{bc}}$ & $54^{\mathrm{cd}}$ & 44 \\
GZT202-7 & Tri5-1/Tri5-4 & $46^{\mathrm{bcd}}$ & $72^{\mathrm{bcd}}$ & 48 \\
GZT202-3 & Tri5-1/Tri5-4 & $53^{\mathrm{bc}}$ & $80^{\mathrm{bc}}$ & 34 \\
GZT202-2 & Tri5-1/Tri5-4 & $42^{\mathrm{cd}}$ & $81^{\mathrm{bc}}$ & 39 \\
GZT202-9 & Tri5-1/Tri5-4 & $17^{\mathrm{cd}}$ & $112^{\mathrm{ab}}$ & 18 \\
GZT40 & Tri5-3 & $1^{\mathrm{e}}$ & $124^{\mathrm{a}}$ & 1 \\
Water & & $0^{\mathrm{e}}$ & $150^{\mathrm{a}}$ & $<1$ \\
\hline
\end{tabular}

*Genotypes as in Table 1.

† Data from greenhouse tests 1 and 2 were combined. Mean percentage of blighted spikelets per ear in the greenhouse tests. Numbers in each column followed by the same letter are not significantly different at $P \leqslant 0 \cdot 05$.

$\ddagger$ Mean kernel weight per 100 ears was $138 \mathrm{~g}$ for test 1 and $161 \mathrm{~g}$ for test 2 for ears inoculated with water. Numbers in each column followed by the same letter are not significantly different at $P \leqslant 0 \cdot 05$.

\Deoxynivalenol was determined as in Table 1.

blighted by mutant GZT40 was 1, 3, 4 and $4 \%$ in tests 2, 3, 4 and 5, respectively. The percentage of spikelets blighted by mutant GZT201-2 was 10,13 and $21 \%$ in tests 3, 4 and 5, respectively. The percentage of spikelets blighted by mutant GZT201-1 was $3 \%$, and by mutant GZT201-5 was $53 \%$, each in a single test. No symptoms of spikelet blight were observed on water-inoculated control plants in any of the five greenhouse tests.

In greenhouse tests 1,2 and 5, virulence was also assessed on the basis of kernel yield loss. In tests 1 and 2, kernels were also analysed for deoxynivalenol content. Strain GZ3639 decreased yields by 78, 88 and $59 \%$ in tests 1, 2 and 5, respectively, and produced deoxynivalenol at 220 and $100 \mu \mathrm{g}$ (g kernel dry wt) ${ }^{-1}$ in the first two tests. Yield loss caused by mutant GZT40 was 17 and $0 \%$ in tests 2 and 5 , respectively. Yield loss caused by mutant GZT201-2 was $30 \%$ in test 5 . Due to greenhouse environmental variation, including poor temperature control and problems with aphids, thrips and mildew, kernel yield data were considerably more variable than spikelet blight data. In tests 3 and 4, seed production was poor, and yield data for these tests are not available.

\section{Effect of the Tri5 complementing vector alone on virulence of the wild-type strain on wheat ears}

Complementation of mutant GZT40 by transformation restored deoxynivalenol production in vitro and in planta but did not restore virulence on wheat ears under field conditions. To test the hypothesis that the complementing vector itself might abolish virulence, vector
pADD6-1 was transformed into the trichotheceneproducing wild-type strain GZ3639. Eighty-three transformants were recovered following transformation of GZ3639 with pADD6-1, followed by selection for growth on acetamide. Nine transformants were selected for further analysis and were reisolated from single conidia. The integration of all or part of the pADD6-1 vector was confirmed by PCR analysis using primers specific for the amdS gene. All nine transformants appeared to be indistinguishable from strain GZ3639 in growth rate, sporulation, pigmentation and morphology on various agar media (data not shown).

Virulence of the nine transformants was compared to virulence of strain GZ3639 and of mutant GZT40 in greenhouse tests 1 and 2, and data from the two tests were combined for statistical analysis and presentation in Table 2. Variability in spikelet blight symptoms, yield loss and deoxynivalenol content was observed among the transformants. However, all nine transformants produced deoxynivalenol in planta, and seven were as virulent as the wild-type strain by one or more parameters tested. Even though some transformants exhibited reduced virulence, the fact that most did not indicates that the low virulence of the Tri5 complemented mutants GZT201-1, GZT201-2 and GZT201-5 was not due to vector pADD6-1 alone.

\section{Genetic analysis of a Tri5-3 mutant}

Because complementation of the disrupted Tri5 did not restore wild-type virulence on wheat ears to mutant GZT40, it is possible that mutant GZT40 differs from 
Table 3. Frequency of heterozygous perithecia and segregation of nit auxotrophy and Tri5 alleles in crosses of transformants to G. zeae strain GZ3639

\begin{tabular}{|c|c|c|c|c|c|c|c|c|c|c|}
\hline \multirow[t]{3}{*}{ Parent $1^{*}$} & \multirow[t]{3}{*}{ Parent $2^{*}$} & \multirow{3}{*}{$\begin{array}{c}\text { Cross } \\
\text { no. }\end{array}$} & \multicolumn{2}{|c|}{ Number of perithecia $\nmid$} & \multicolumn{6}{|c|}{ Number of progeny $\ddagger$} \\
\hline & & & \multirow[t]{2}{*}{ Total } & \multirow[t]{2}{*}{ Heterozygous } & \multirow[t]{2}{*}{ Total } & \multirow[t]{2}{*}{$n i t^{+}$} & \multicolumn{4}{|c|}{ Tri5 allele } \\
\hline & & & & & & & 1 & 3 & $1 / 4$ & $3 / 4$ \\
\hline \multirow[t]{7}{*}{ GZ3639A (Tri5-1) } & GZT40A (Tri5-3) & 3 & 14 & 3 & 19 & 5 & 10 & 9 & & \\
\hline & & & & & 18 & 2 & 7 & 10 & & \\
\hline & & & & & 9 & 1 & 4 & 5 & & \\
\hline & GZT40B (Tri5-3) & 4 & 6 & 1 & 24 & 4 & 18 & 6 & & \\
\hline & GZT201-2A (Tri5-3/Tri5-4) & 12 & 7 & 1 & 24 & 13 & 21 & 0 & 0 & 3 \\
\hline & GZT201-2B (Tri5-3/Tri5-4) & 13 & 5 & 2 & 24 & 11 & 17 & 0 & 0 & 7 \\
\hline & & & & & 24 & 10 & 21 & 0 & 0 & 3 \\
\hline GZ3639B (Tri5-1) & GZT201-2A (Tri5-3/Tri5-4) & 10 & 6 & 1 & 23 & 1 & 3 & 0 & 2 & 18 \\
\hline
\end{tabular}

*Genotypes as in Table 1. Strain GZ3639A is a nit3 mutant, strain GZT201-2A is a NitM mutant, and strains GZ3639B, GZT40A, GZT40B and GZT201-2B are nit1 mutants.

†Total number of perithecia isolated; number of heterozygous perithecia.

$\ddagger$ Total number of progeny isolated from each heterozygous perithecium; number of prototrophic progeny; number of progeny with each Tri5 allele.

its progenitor strain, GZ3639, at virulence loci in addition to Tri5. To test whether reduced virulence was due solely to Tri5 gene disruption, a genetic cross was performed between GZT40 and GZ3639. Because G. zeae is self-fertile, nitrate-nonutilizing (nit) mutants were used as parents to facilitate identification of heterozygous perithecia. Preliminary tests indicated that nit mutants of strain GZ3639 retained wild-type virulence on wheat ears (data not shown).

The results of genetic crosses of two independent nit 1 mutants of strain GZT40 and a nit 3 mutant of strain GZ3639 are presented in Table 3. Progeny from 20 perithecia formed at the colony interfaces were analysed for nitrate utilization, and putative heterozygous perithecia were then analysed for segregation of Tri5 alleles. The frequency of heterozygous perithecia at the interface was $21 \%$ for cross 3 and $17 \%$ for cross 4 . Among the four heterozygous perithecia from crosses of strains GZT40 and GZ3639, 58 nit mutants and 12 prototrophic progeny were recovered. This ratio was not significantly different from the expected $3: 1$ ratio $\left(\chi^{2}\right.$ $=2.69, P=0.10)$. Among the 69 progeny analysed for Tri5 alleles, 39 progeny had the Tri5-1 allele and 30 progeny had the Tri5-3 allele. This ratio was not significantly different from the expected $1: 1$ ratio $\left(\chi^{2}=\right.$ $0 \cdot 93, P=0 \cdot 34)$.

The association of virulence with Tri5 alleles was investigated by comparing the ability of parental strains and random ascospore progeny to cause wheat ear blight in greenhouse tests 1 and 2 . The virulence data were combined for statistical analysis and presentation in Table 4. Progeny from each cross are ranked relative to the parents and to other progeny from the same cross for percentage of blighted spikelets, yield loss and deoxynivalenol content. Results were consistent with cosegregation of higher virulence with the wild-type Tri5-1 allele, and of lower virulence with the disrupted Tri5-3 allele. In cross 3, Tri5-1 progeny averaged $65 \pm 27 \%$ blighted spikelets, and $60 \pm 15 \%$ yield loss; whereas Tri5-3 progeny averaged $1 \%$ blighted spikelets and $3 \%$ yield loss. In cross 4, Tri5-1 progeny averaged $94 \pm 10 \%$ blighted spikelets and $78 \pm 10 \%$ yield loss; whereas Tri5-3 progeny averaged $1 \%$ blighted spikelets and $34 \pm 14 \%$ yield loss. In cross 4 , Tri5-3 progeny 4-1, 4-4 and 4-23 showed low virulence based on spikelet blight, but moderate virulence based on yield loss. The low yield in the absence of blight symptoms is unexplained, and may reflect greenhouse environmental variation. Recombinant trichothecene-producing Tri5-1 progeny with the low virulence of their Tri5-3 parent GZT40 were not recovered in cross 3 or cross 4 . These observations indicate that the low virulence of the Tri5 complemented mutants GZT201-1, GZT201-2 and GZT201-5 is not likely to be due to mutations of strain GZT40 at additional loci that affect virulence.

\section{Genetic analysis of a Tri5-3/Tri5-4 complemented mutant}

Two rounds of transformation were employed to generate the complemented mutants: the first round to disrupt Tri5 and the second round to add a functional Tri5 back to the disruption mutant GZT40. Since the reduced virulence of GZT40 is not due to non-target effects of transformation (see previous section), it is possible that the reduced virulence of the complemented mutants is due to spontaneous mutation or other nontarget effects of the second round of transformation. To analyse reduced virulence of the Tri5 complemented 
Table 4. Random ascospore analysis of segregation of the Tri5-1 and Tri5-3 alleles and of virulence on wheat ears inoculated in the greenhouse

\begin{tabular}{|c|c|c|c|c|c|c|c|c|}
\hline \multirow[t]{2}{*}{ Genotype } & \multicolumn{4}{|c|}{ Cross 3} & \multicolumn{4}{|c|}{ Cross 4} \\
\hline & Strain & $\begin{array}{c}\text { Blighted } \\
\text { spikelets } \\
(\%) \dagger\end{array}$ & $\begin{array}{l}\text { Yield } \\
(\mathrm{g}) \neq\end{array}$ & $\begin{array}{l}\text { Deoxynivalenol } \\
\quad\left(\mu \mathrm{g} \mathrm{g}^{-1}\right) \mathbb{S}\end{array}$ & Strain & $\begin{array}{c}\text { Blighted } \\
\text { spikelets } \\
(\%) \dagger\end{array}$ & $\begin{array}{l}\text { Yield } \\
(\mathrm{g}) \neq\end{array}$ & $\begin{array}{l}\text { Deoxynivalenol } \\
\quad\left(\mu \mathrm{g} \mathrm{g}^{-1}\right) \mathbb{S}\end{array}$ \\
\hline \multirow[t]{10}{*}{ Tri5-1 } & GZ3639 & $92^{\mathrm{a}}$ & $35^{\mathrm{c}}$ & 160 & GZ3639 & $92^{\mathrm{a}}$ & $35^{\mathrm{c}}$ & 160 \\
\hline & $3-4$ & $100^{\mathrm{a}}$ & $45^{\mathrm{c}}$ & 200 & $4-8$ & $100^{\mathrm{a}}$ & $21^{\mathrm{c}}$ & 300 \\
\hline & $3-8$ & $97^{\mathrm{a}}$ & $32^{\mathrm{c}}$ & 170 & $4-9$ & $100^{\mathrm{a}}$ & $22^{\mathrm{c}}$ & 280 \\
\hline & $3-14$ & $96^{\mathrm{a}}$ & $32^{\mathrm{c}}$ & 160 & $4-6$ & $100^{\mathrm{a}}$ & $22^{\mathrm{c}}$ & 260 \\
\hline & $3-10$ & $56^{\mathrm{b}}$ & $45^{\mathrm{c}}$ & 91 & $4-5$ & $100^{\mathrm{a}}$ & $30^{\mathrm{c}}$ & 240 \\
\hline & $3-15$ & $61^{\mathrm{b}}$ & $62^{\mathrm{c}}$ & 84 & $4-10$ & $86^{\mathrm{a}}$ & $50^{\mathrm{c}}$ & 75 \\
\hline & $3-19$ & $58^{\mathrm{b}}$ & $74^{\mathrm{c}}$ & 74 & $4-7$ & $75^{\mathrm{b}}$ & $54^{\mathrm{c}}$ & 93 \\
\hline & $3-17$ & $55^{\mathrm{b}}$ & $75^{\mathrm{c}}$ & 55 & & & & \\
\hline & $3-9$ & $31^{\mathrm{b}}$ & $72^{\mathrm{c}}$ & 38 & & & & \\
\hline & $3-13$ & $31^{\mathrm{b}}$ & $100^{\mathrm{a}}$ & 39 & & & & \\
\hline \multirow[t]{11}{*}{ Tri5-3 } & $3-6$ & $1^{\mathrm{c}}$ & $94^{\mathrm{b}}$ & $<1$ & $4-4$ & $1^{\mathrm{c}}$ & $51^{\mathrm{c}}$ & $<1$ \\
\hline & $3-16$ & $1^{\mathrm{c}}$ & $126^{\mathrm{a}}$ & $<1$ & $4-1$ & $1^{\mathrm{c}}$ & $75^{\mathrm{b}}$ & $<1$ \\
\hline & $3-7$ & $1^{\mathrm{c}}$ & $129^{a}$ & $<1$ & $4-23$ & $1^{\mathrm{c}}$ & $80^{\mathrm{b}}$ & $<1$ \\
\hline & $3-3$ & $1^{\mathrm{c}}$ & $144^{\mathrm{a}}$ & $<1$ & $4-2$ & $1^{\mathrm{c}}$ & $128^{\mathrm{a}}$ & $<1$ \\
\hline & $3-5$ & $1^{\mathrm{c}}$ & $147^{\mathrm{a}}$ & $<1$ & $4-16$ & $1^{\mathrm{e}}$ & $156^{\mathrm{a}}$ & $<1$ \\
\hline & $3-11$ & $1^{\mathrm{c}}$ & $152^{\mathrm{a}}$ & $<1$ & $4-3$ & $1^{\mathrm{c}}$ & $164^{\mathrm{a}}$ & $<1$ \\
\hline & $3-12$ & $1^{\mathrm{c}}$ & $158^{\mathrm{a}}$ & $<1$ & & & & \\
\hline & $3-18$ & $1^{\mathrm{c}}$ & $171^{\mathrm{a}}$ & $<1$ & & & & \\
\hline & $3-1$ & $1^{\mathrm{c}}$ & $188^{\mathrm{a}}$ & $<1$ & & & & \\
\hline & GZT40 & $1^{\mathrm{c}}$ & $128^{\mathrm{a}}$ & 1 & GZT40 & $1^{\mathrm{c}}$ & $128^{\mathrm{a}}$ & 1 \\
\hline & Water & $0^{\mathrm{c}}$ & $150^{\mathrm{a}}$ & $<1$ & Water & $0^{\mathrm{c}}$ & $150^{\mathrm{a}}$ & $<1$ \\
\hline
\end{tabular}

*,†,‡, $\$$ Footnotes as in Table 2. Parents GZ3639 and GZT40 of crosses 3 and 4 are described in Table 3. In the blighted spikelets columns, numbers followed by the letter a are different from parent GZT40, but not from parent GZ3639; those followed by the letter b are different from both GZ3639 and GZT40; those followed by the letter c are different from GZ3639, but not from GZT40. In the yield columns, numbers followed by a are different from GZ3639, but not from GZT40; those followed by b are different from both GZ3639 and GZT40; and those followed by $\mathrm{c}$ are different from GZT40, but not from GZ3639. Significance levels $P \leqslant 0 \cdot 05$.

mutants, mutants GZT201-1, GZT201-2 and GZT2015 were crossed with the wild-type strain GZ3639. Again, nitrate-nonutilizing mutants were used as parents to facilitate identification of heterozygous perithecia. Forty-seven perithecia from crosses with strains GZT201-1 and GZT201-5 were isolated and analysed, but none were heterozygous (data not shown). From crosses between strains GZT201-2 and GZ3639, heterozygous perithecia were recovered at an overall frequency of $16 \%$ (Table 3). Among the four heterozygous perithecia obtained from these parents, 60 nit mutants and 35 prototrophic progeny were recovered. This ratio deviated significantly from the expected $3: 1$ ratio $\left(\chi^{2}=5 \cdot 4, P<0 \cdot 05\right)$. All progeny were also analysed for the presence of the Tri5-1 allele inherited from parent GZ3639 and the Tri5-3 and Tri5-4 alleles inherited from parent GZT201-2. Segregation of Tri5-1 and Tri5-3 alleles deviated significantly from the expected $1: 1$ ratio $\left(\chi^{2}=10 \cdot 7, P<0 \cdot 01\right)$. Previously, PCR and Southern blot analyses indicated that in strain GZT201-2, integration of vector pADD6-1 occurred ectopically rather than at the Tri5 locus (Proctor et al.,
1997). If the locus where Tri5-4 integrated is not closely linked to the native Tri5 locus, then Tri5-3 and Tri5$1 /$ Tri5-4 recombinant progeny should be expected. Two Tri5-1/Tri5-4 progeny were obtained, which confirmed that pADD6-1 did integrate outside the Tri5 locus.

The reduced virulence of strain GZT201-2 might have arisen by insertion of vector pADD6-1 into a virulence locus or by spontaneous mutation of a virulence gene, or genes, during the transformation process. Perfect cosegregation of the Tri5-4 allele with low virulence would indicate that reduced virulence was caused by insertion of pADD6-1 into a virulence locus. On the other hand, recovery of progeny with the Tri5-1 allele and low virulence, or of progeny with the Tri5-4 allele and wild-type virulence, would indicate a mutation at another site. The association of virulence with Tri5 alleles was investigated by comparing the ability of parental strains and 47 random ascospore progeny to cause wheat ear blight in greenhouse test 4 . In Table 5, progeny from each cross are ranked relative to the parents and to other progeny from the same cross for 
Table 5. Random ascospore analysis of segregation of Tri5-1, Tri5-3 and Tri5-4 alleles and of virulence on wheat ears inoculated in the greenhouse

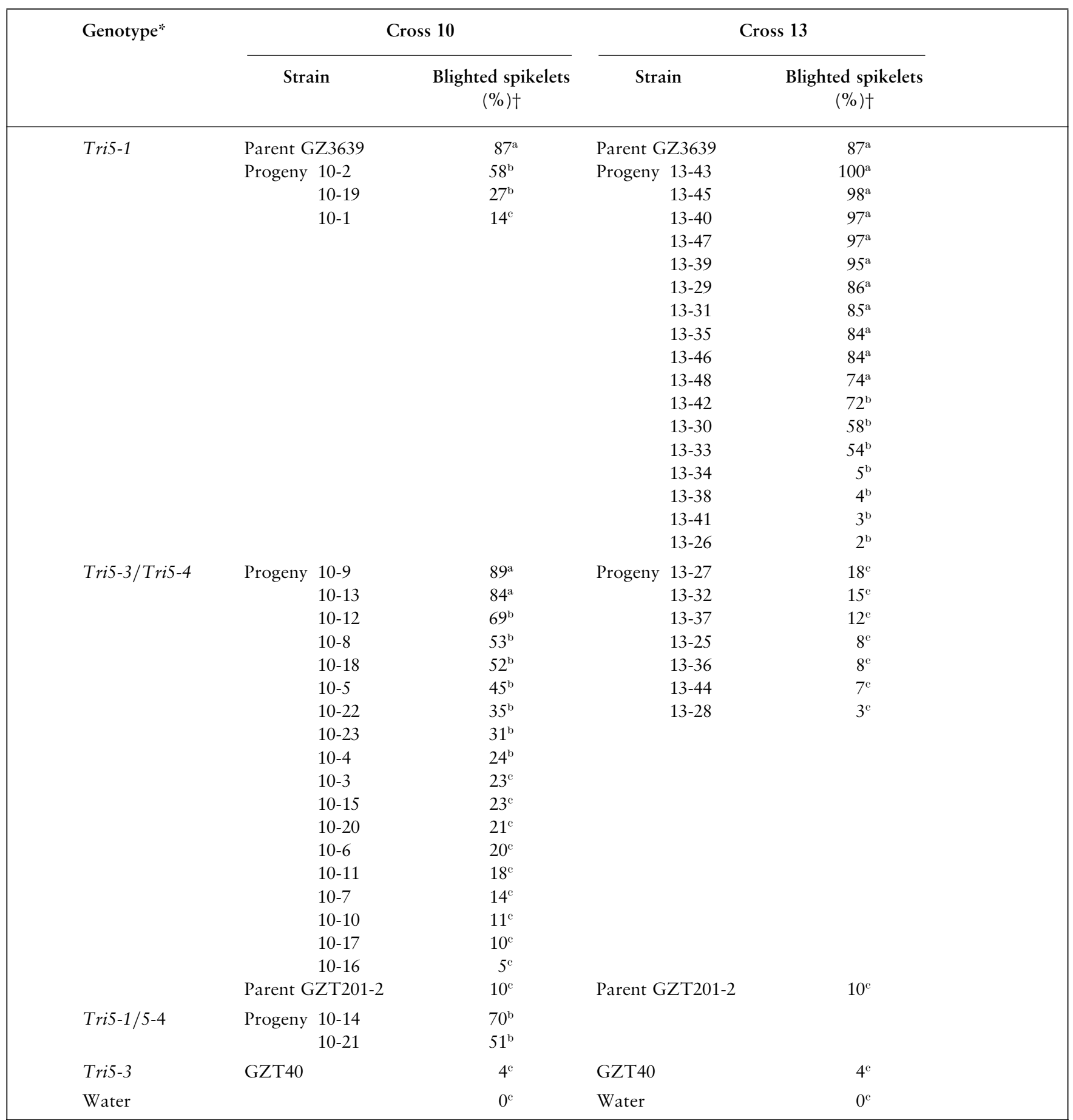

*,†Footnotes as in Table 2, except that data are from greenhouse test 4. Parents GZ3639 and GZT201-2 of crosses 10 and 13 are described in Table 3. In each column, numbers followed by the letter a are different from parent GZT201-2, but not from parent GZ3639; those followed by the letter b are different from both GZ3639 and GZT201-2; those followed by the letter c are different from GZ3639, but not from GZT201-2; significance level $P \leqslant 0 \cdot 05$.

percentage of blighted spikelets. Several recombinant progeny with low virulence but without the Tri5-3 or Tri5-4 allele were recovered. Furthermore, several recombinant progeny with the Tri5-3 and Tri5-4 alleles and with wild-type or near wild-type virulence also were recovered. In greenhouse test 5 , the moderate to high virulence of six of these recombinant Tri5-3/Tri5-4 progeny was confirmed (data not shown). These obser- 
vations indicate that the low virulence of strain GZT201-2 is not likely to have been caused by insertion of vector pADD6-1 into a virulence locus.

\section{Phenotypic variation among mutant strains}

On a range of agar culture media, strain GZ3639 and the majority of wild-type strains of G. zeae produce rapid, profuse mycelial growth, with a carmine red undersurface. As the cultures age, mycelia can become tan, yellow or pink, and macroconidia develop. On carrot agar, perithecia with ascospores can develop. The GZT40 mutant, the GZT201 complemented mutants, and the GZT202 transformants produced the profuse mycelium, carmine red undersurface, macroconidia and perithecia typical of the wild-type strain. However, the radial growth rate of the three GZT201 complemented mutants was significantly slower than that of the wildtype strain and mutant GZT40 from which they were derived. On plates of minimal medium, for example, radial growth rates (mean \pm standard deviation, $\mathrm{mm} \mathrm{d}^{-1}$ ) were $10 \cdot 1 \pm 0 \cdot 1,9 \cdot 8 \pm 0 \cdot 8$ and $9 \cdot 3 \pm 0 \cdot 6$ for mutants GZT201-1, GZT201-2 and GZT201-5, respectively. In contrast, radial growth rates of the wild-type strain GZ3639, mutant GZT40 and the GZT202 transformants ranged from $12 \cdot 5$ to $14 \cdot 0 \mathrm{~mm} \mathrm{~d}^{-1}$.

\section{DISCUSSION}

Mutants that are blocked or altered in toxin biosynthesis are standard tools for rigorously testing the role of toxins in plant pathogenesis. Toxin-nonproducing mutants are particularly useful for analysis of mycotoxins and other nonselective fungal toxins that can affect a wide range of organisms, and whose role in plant disease has been controversial. Mutants altered in biosynthesis of trichothecene mycotoxins have been obtained by UV light mutagenesis or Tri5 gene disruption in Fusarium sporotrichioides, Gibberella pulicaris and G. zeae (Desjardins \& Hohn, 1997). Laboratory, greenhouse and field tests of these mutants have shown that trichothecenes are not important for the virulence of G. pulicaris on potato tubers. Trichothecenes are, however, important for the virulence of $F$. sporotrichioides and G. pulicaris on parsnip root, and for the virulence of G. zeae on wheat seedlings, wheat ears and maize ears (Desjardins \& Hohn, 1997; Harris et al., 1999).

Although changes in virulence following transformation-mediated disruption or complementation of toxin genes are strong evidence that a toxin is important in pathogenesis, the transformation process itself has the potential to cause alterations at loci other than the targeted gene (Oliver \& Osbourn, 1995). In the present study, non-target effects of transformation were investigated by (1) evaluating a number of transformants in which the complementing vector inserted ectopically, and (2) outcrossing a disruption mutant and a complemented mutant to the wild-type strain from which they were derived, and then analysing the progeny for cosegregation of the introduced genes and virulence on wheat ears. Our results indicate that strains of G. zeae can undergo spontaneous or transformation-induced mutations that significantly reduce virulence on wheat ears in both greenhouse and field tests.

Virulence assays are very complex, and have several critical steps, each of which can be a significant source of error. Although some biological variation is unavoidable, levels of virulence of trichothecene mutants of $G$. zeae on wheat ears have usually correlated with levels of virulence on wheat seedlings, when results of different experiments and from different laboratories have been compared (Proctor et al., 1995, 1997; Desjardins et al., 1996; Nicholson et al., 1998). Trichothecenenonproducing mutants have been low in virulence, and trichothecene-producing revertants have been high in virulence, on wheat seedlings, wheat ears and maize ears (Proctor et al., 1995, 1997; Desjardins et al., 1996; Eudes et al., 1998; Nicholson et al., 1998; Harris et al., 1999). On the other hand, trichothecene-producing complemented mutants were restored to moderate to high virulence on wheat seedlings in a previous study (Proctor et al., 1997), but were not consistently restored to moderate or high virulence on wheat ears in the present study. The reasons for the discrepancy between these two virulence assays are not known. Previous surveys of wheat have concluded that ear blight resistance and seedling blight resistance are correlated in some genotypes but not in others (Parry et al., 1995). All modern breeding programmes for wheat ear blight resistance use ear inoculation tests almost exclusively (Bai \& Shaner, 1994).

The mechanisms for the reduced virulence of the Tri5complemented transformants on wheat ears are not known. All three transformants were restored to production of high levels of deoxynivalenol by transformation-mediated complementation with Tri5. In addition to trichothecenes, G. zeae produces a wide variety of biologically active metabolites, including fusarins, naphthazarins and zearalenone (De Nijs et al., 1996), and mutations that affect their production might affect virulence. However, none of these metabolites has been shown to play a role in wheat pathogenesis. In addition, loss of virulence on wheat ears was not associated with obvious changes in strain morphology, sporulation or sexual fertility. Complemented mutants GZT201-1, GZT201-2 and GZT201-5 showed a slightly reduced radial growth rate on agar media, but were otherwise indistinguishable from the wild-type strain, GZ3639. Our study cannot exclude the possibility of more subtle effects on pathogen fitness. However, all of the mutants could be reisolated at high frequencies from kernels harvested from the field $35 \mathrm{~d}$ after inoculation, indicating that the strains were able to survive in wheat ears under field conditions, even though they produced few symptoms of disease.

Genetic instability is a widely reported trait in the genus Fusarium (Nelson et al., 1981), and our results indicate that genetic instability is likely to occur in G. zeae. Thus 
gene disruption experiments in this species should be interpreted with caution, particularly those involving putative virulence genes. In principle, the availability of a system for sexual recombination in G. zeae should facilitate the characterization of mutants generated by gene disruption or by other methods. In practice, however, genetic analysis in G. zeae is still rather laborious due to the need to use marked strains to identify heterozygous perithecia. In addition, the frequency of heterozygous perithecia is relatively low, ranging from 0 to $35 \%$ in a previous study (Bowden \& Leslie, 1999) and from 0 to $21 \%$ in our study. Nevertheless, using this approach, we were able to genetically dissect the aberrant virulence we observed following transformation-mediated complementation of a Tri5 disruption mutant. We also were able to confirm our previous findings that trichothecenes are important virulence factors in wheat ear blight. Thus sexual recombination in G. zeae has the potential to facilitate the characterization of a wide range of mutants and the identification of genes that play important roles in the economically important diseases caused by this pathogen.

\section{ACKNOWLEDGEMENTS}

We thank R. Bowden, Kansas State University, Manhattan, KS, for providing a fungal mutant and the crossing method prior to its publication, S. Folmar, M. Moore, H. Roseman and T. Wilson for technical assistance, D. Schisler and D. Palmquist for assistance with statistical analysis, and $M$. Giovannini for assistance in the greenhouse tests.

Disclaimer: Names are necessary to report factually on available data; however, the USDA neither guarantees nor warrants the standard of the products, and the use of the name by USDA implies no approval of the product to the exclusion of others that may also be suitable.

\section{REFERENCES}

Bai, G.-H. \& Shaner, G. (1994). Scab of wheat: prospects for control. Plant Dis 78, 760-766.

Bai, G.-H. \& Shaner, G. (1996). Variation in Fusarium graminearum and cultivar resistance to wheat scab. Plant Dis $\mathbf{8 0}$, 975-979.

Bowden, R. L. \& Leslie, J. F. (1992). Nitrate-nonutilizing mutants of Gibberella zeae (Fusarium graminearum) and their use in determining vegetative compatibility. Exp Mycol 16, 308-315.

Bowden, R. L. \& Leslie, J. F. (1999). Sexual recombination in Gibberella zeae. Phytopathology 89, 182-188.

Correll, J. C., Klittich, C. J. R. \& Leslie, J. F. (1987). Nitrate nonutilizing mutants of Fusarium oxysporum and their use in vegetative compatibility tests. Phytopathology 77, 1640-1646.

De Nijs, M., Rombouts, F. \& Notermans, S. (1996). Fusarium molds and their mycotoxins. J Food Saf 16, 15-58.
Desjardins, A. E. \& Hohn, T. M. (1997). Mycotoxins in plant pathogenesis. Mol Plant-Microbe Interact 10, 147-152.

Desjardins, A. E., Hohn, T. M. \& McCormick, S. P. (1993). Trichothecene biosynthesis in Fusarium species: chemistry, genetics, and significance. Microbiol Rev 57, 595-604.

Desjardins, A. E., Proctor, R. H., Bai, G., McCormick, S. P., Shaner, G., Buechley, G. \& Hohn, T. M. (1996). Reduced virulence of trichothecene antibiotic-nonproducing mutants of Gibberella zeae in wheat field tests. Mol Plant-Microbe Interact 9, 775-781.

Eudes, F., Collin, J., Rioux, S. \& Comeau, A. (1998). The trichothecenes, a major component of wheat head scab pathogenesis. In Abstracts of the National Fusarium Head Blight Forum, p. 67. St Paul, MN: University of Minnesota Extension Service.

Harris, L. J., Desjardins, A. E., Plattner, R. D., Nicholson, P., Butler, G., Young, J. C., Weston, G., Proctor, R. H. \& Hohn, T. M. (1999). Possible role of trichothecene mycotoxins in virulence of Fusarium graminearum on maize. Plant Dis 83, 954-960.

Marasas, W. F. O., Nelson, P. E. \& Toussoun, T. A. (1984). Toxigenic Fusarium Species - Identity and Mycotoxicology. University Park, PA: Pennsylvania State University Press.

Nelson, P. E., Toussoun, T. A. \& Cook, R. J. (1981). Fusarium: Diseases, Biology, and Taxonomy. University Park, PA: Pennsylvania State University Press.

Nelson, P. E., Toussoun, T. A. \& Marasas, W. F. O. (1983). Fusarium Species - an Illustrated Manual for Identification. University Park, PA: Pennsylvania State University Press.

Nicholson, P., Simpson, D. R., Weston, G., Rezanoor, H. N., Lees, A. K., Parry, D. W. \& Joyce, D. (1998). Detection and quantification of Fusarium culmorum and Fusarium graminearum in cereals using PCR assays. Physiol Mol Plant Pathol 53, 17-37.

Oliver, R. \& Osbourn, A. (1995). Molecular dissection of fungal phytopathogenicity. Microbiology 141, 1-9.

Parry, D. W., Jenkinson, P. \& McLeod, L. (1995). Fusarium ear blight (scab) in small grain cereals - a review. Plant Pathol 44, 207-238.

Proctor, R. H., Hohn, T. M. \& McCormick, S. P. (1995). Reduced virulence of Gibberella zeae caused by disruption of a trichothecene toxin biosynthetic gene. Mol Plant-Microbe Interact 8, 593-601.

Proctor, R. H., Hohn, T. M. \& McCormick, S. P. (1997). Restoration of wild-type virulence to Tri5 disruption mutants of Gibberella zeae via gene reversion and mutant complementation. Microbiology 143, 2583-2591.

Schwadorf, K. \& Muller, H.-M. (1989). Determination of ergosterol in cereals, mixed feed components, and mixed feeds by liquid chromatography. J Assoc Off Anal Chem 72, 457-462.

Steel, R. G. D. \& Torrie, J. H. (1980). Principles and Procedures of Statistics, a Biometrical Approach, 2nd edn. New York: McGraw-Hill.

Sutton, J. C. (1982). Epidemiology of wheat head blight and maize ear rot caused by Fusarium graminearum. Can J Plant Pathol 4, 195-209.

Received 21 January 2000; revised 11 April 2000; accepted 27 April 2000. 